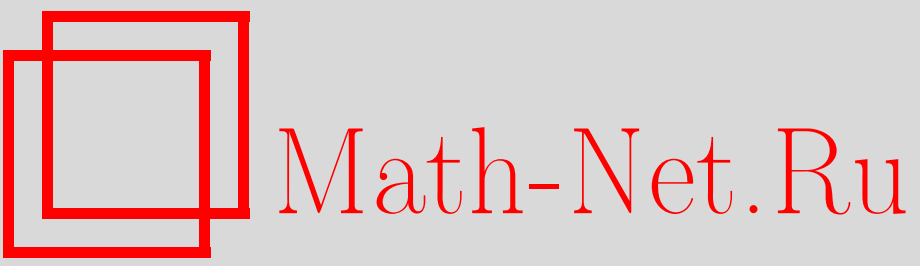

С. Босколо, С. А. Деревянко, С. К. Турицын, А. С. Ковалев, М. М. Богдан, Эволюция оптических импульсов в волоконных линиях связи с концентрированными нелинейными устройствами как задача об отображении, $T M \Phi, 2005$, том 144, номер 2, 277-289

DOI: https://doi.org/10.4213/tmf1852

Использование Общероссийского математического портала Math-Net.Ru подразумевает, что вы прочитали и согласны с пользовательским соглашением

http: //www. mathnet.ru/rus/agreement

Параметры загрузки:

IP: 54.162 .27 .143

26 апреля 2023 г., 16:12:37 


\section{ЭВОЛЮЦИЯ ОПТИЧЕСКИХ ИМПУЛЬСОВ В ВолОКОНнЫХ ЛИнИЯх СВЯЗИ С КОНЦЕНТРИРОВАННЫМИ НЕЛИНЕЙНЫМИ УСТРОЙСТВАМИ КАК ЗАДАЧА ОБ ОТОБРАЖЕНИИ}

\footnotetext{
Исследована проблема передачи информации в высокоскоростных волоконных линиях связи с периодически распределенными нелинейными оптическими устройствами. Впервые, по нашему мнению, построена теоретическая модель, описывающая квазилинейный режим распространения импульса в системе с нелинейными точечными элементами. Сформулирована задача отображения при распространении импульса вдоль одной секции и показано, что в частном случае применения нелинейных оптических петлеобразных зеркал характеристики импульса в устоявшемся состоянии, предсказываемые теорией, достаточно точно совпадают с результатами прямых численных
} расчетов.

Ключевые слова: дисперсионные системы с точечной нелинейностью, задача отображения, автосолитоны.

\section{1. ВВЕДЕНИЕ}

Солитоны в оптическом волокне возникают благодаря балансу двух физических эффектов: расплывания импульса из-за дисперсии групповой скорости и нелинейной фокусировки, возникающей вследствие зависимости показателя преломления волокна от интенсивности сигнала. Обычные солитоны [1] (т.е. солитоны, распространяюшиеся в волокнах с однородной дисперсией) и солитоны в среде с управляемой дисперсией (УД) [2], [3] (т.е. солитоны, распространяюшиеся в волокнах с периодически меняющейся дисперсией) были предметом интенсивных исследований в течение последних десятилетий ввиду их возможных применений в качестве носителей информации в оптических коммуникационных системах. Однако задача модификации сушествуюших наземных

*Photonics Research Group, School of Engineering and Applied Science, Aston University, Birmingham B4 7ET, United Kingdom. E-mail: boscolsa@aston.ac.uk, s.derevyanko@aston.ac.uk, s.k.turitsyn@aston.ac.uk

† Физико-технический институт низких температур им. Б. И. Веркина НАН Украины, Харьков 61103, Украина. E-mail: kovalev@ilt.kharkov.ua, bogdan@ilt.kharkov.ua 
оптоволоконных коммуникаций с целью достижения более высокой плотности передаваемой информации приводит, вообше говоря, к необходимости использования более коротких импульсов и соответственно к необходимости контроля над сильными дисперсионными эффектами (математическое определение мощности дисперсионного отображения можно найти, например, в [3]), имеющими место далеко за пределами той области, где наблюдалось стабильное распространение УД-солитонов [4]. В режиме сильного дисперсионного отображения, который обычно называется квазилинейным режимом, несушие импульсы периодически испытывают большие временны́е уширения, и, следовательно, их интенсивность быстро изменяется при движении вдоль волокна, а потому аккумулированньй эффект, создаваемый мгновенной нелинейностью, должен будет усредняться. Эта схема в некотором смысле противоположна сценарию распространения обычного солитона или УД-солитона, в котором нелинейность волокна играет важную роль для сохранения формы импульсов в процессе их прохождения.

Известно, что искажения оптических сигналов в волоконной коммуникационной системе можно уменьшить, используя регенерируюшие устройства. В последнее время было установлено, что периодическое размешение в линии передачи нелинейных оптических устройств (НОУ), таких как нелинейные оптические петлеобразные зеркала (НОПЗ), полупроводниковые насышаемые поглотители и полупроводниковые амплитудные усилители, является эффективным способом решения проблемы регенерации оптических сигналов [5]-[7], при этом удается достигнуть стабильного распространения импульса и практически неограниченной дальности передачи информации в высокоскоростных системах с сильной УД [6]. В работах [5], [6] было показано численно, что при определенных условиях взаимодействие между дисперсией в волокне, локальной нелинейностью, обеспечиваемой встроенными НОПЗ, и действием элементов линейного контроля типа оптических фильтров приводит к образованию автосолитонов, периодически воспроизводимых на выходе каждого сегмента линии передачи. Термин "автосолитоны" здесь используется для обозначения устойчивых локализованных импульсов с параметрами, определяемыми свойствами системы, которые возникают в моделях, сочетающих консервативные и диссипативные, дисперсионные и нелинейные члены [8].

В данной работе впервые предлагается теория, описывающая передачу сигнала в УД-волоконных коммуникационных системах в квазилинейном режиме с периодически встроенными точечными НОУ. Сформулирована математическая проблема отображения для прохождения импульса через единичный сегмент линии передачи. Без потери обшности в качестве конкретных реализаций НОУ рассмотрены усиливаюшие устройства, работающие в соответствии со степеннь́м законом преобразования амплитуды сигнала (power-law devices, или УСЗ) и НОПЗ. В случае УСЗ удается построить аналитическое решение проблемы отображения в стационарных точках в некоторых специальных предельных случаях. В случае НОПЗ для нахождения характеристик сташионарного движения импульса оказьвается применим вариационный метод, и можно показать, что теория достаточно хорошо воспроизводит особенности, наблюдаемые во всех случаях численного моделирования этого процесса. 


\section{2. ОСНОВНАЯ МОДЕЛЬ}

Рассматривается эволюция оптического импульса каскадной линии связи с периодическими изменениями дисперсии и нелинейности, с частотной фильтрацией и с НОУ. В этом случае распространение импульса описывается обобщенным нелинейным уравнением Шредингера

$$
i \frac{\partial E}{\partial z}-\frac{1}{2} \beta_{2}(z) \frac{\partial^{2} E}{\partial t^{2}}+\sigma(z)|E|^{2} E=i G\left(z,|E|^{2}\right) E,
$$

где $E(z, t)$ - медленно меняющаяся огибающая импульса в сопутствующей системе координат; $\beta_{2}(z)$ задает изменение дисперсии групповой скорости, обусловленное компенсацией дисперсии, и предполагается, что это периодическая функция от $z$ с периодом $L, \beta_{2}(z)=\beta_{2}(z+L) ; \sigma-$ коэффищиент нелинейности волокна. Обычно величина $\beta_{2}$ выражается через соответствуюший параметр дисперсии $D$ с помощњю формулы $\beta_{2}=-\lambda^{2} D /\left(2 \pi c_{0}\right)$, где $\lambda$ - длина волны несущего сигнала, $c_{0}$ - скорость света, а величина $D$ измеряется в [пкс/(нм·км)]. Функция $G\left(z,|E|^{2}\right)$ описьвает ослабление сигнала за счет потерь в волокне, усиление сигнала с помощью оптических усилителей, действие фильтров и нелинейные добавки, связанные с НОУ, и может быть представлена в следуюшем виде:

$$
\begin{aligned}
G\left(z,|E|^{2}\right)= & -\gamma(z)+\sum_{k} \delta\left(z-k Z_{\mathrm{a}}\right)\left\{\exp \left[\int_{(k-1) Z_{\mathrm{a}}}^{k Z_{\mathrm{a}}} d z \gamma(z)\right]-1\right\}+ \\
& +\sum_{k} \delta\left(z-k Z_{\mathrm{f}}\right)[h(t) *-1]+\sum_{k} \delta\left(z-k Z_{0}\right)\left[f\left(|E|^{2}\right)-1\right]
\end{aligned}
$$

где $h(t)=\mathcal{F}^{-1}[\hat{h}(\omega)]$ - обратное преобразование Фурье от функции пропускания фильтра, а символ $*$ обозначает свертку Фурье. В выражении (2) предполагается, что усилители, фильтры и НОУ размешаются в системе периодически с соответствующими периодами $Z_{\mathrm{a}}, Z_{\mathrm{f}}$ и $Z_{0}$. Члены с дельта-функциями являются компактной записью условий скачков для функции в точках расположения усилителей, фильтров и НОУ, причем подразумевается, что дельта-функции отбирают для произвольной функции $q(E)$ ее значения с левой стороны, т.е.

$$
\int_{k Z_{\beta}^{-}}^{k Z_{\beta}^{+}} d z \delta\left(z-k Z_{\beta}\right) q(E)=q\left(E\left(k Z_{\beta}^{-}, t\right)\right),
$$

где введено обозначение $Z_{\beta}^{ \pm}=Z_{\beta} \pm \epsilon$ в пределе $\epsilon \rightarrow 0$. Величина $\gamma=0.05 \ln (10) \alpha$ представляет собой показатель потерь в волокне, который описывает ослабление сигнала на участке волокна, простираюшегося до вставки $k$-го усилителя, $\alpha$ измеряется в единицах дБ/км, а

$$
\exp \left[\int_{(k-1) Z_{\mathrm{a}}}^{k Z_{\mathrm{a}}} d z \gamma(z)\right]-1
$$

- коэффициент усиления на участке волокна между $(k-1)$-м и $k$-м усилителями. НОУ описываются своими коэффишиентами усиления $f(P)$, зависяшими от мошности. Здесь 
и в дальнейшем упор делается на УСЗ и НОПЗ с несбалансированными потерями (усилением). Функцию переноса в таких системах можно представить в виде

$$
f(P)= \begin{cases}a P^{s} & \text { для УСЗ, } \\ a \sin (b P) e^{i c P} & \text { для НОПЗ, }\end{cases}
$$

при этом $s \in \mathbb{R}, a, b, c \in \mathbb{R}^{+}$суть некоторые заданные постоянные величины.

\section{3. РЕЖИМ ЛИНЕЙНОГО РАСПРОСТРАНЕНИЯ И ЗАДАЧА ОТОБРАЖЕНИЯ}

Чтобы упростить полную модель, задаваемую системой (1), можно сделать несколько физически оправданных предположений. В данном разделе проводится анализ случая линейного распространения импульса в волокне, в котором можно пренебречь нелинейным членом в выражении (1). Такой режим распространения отвечает случаю, когда длина нелинейности $L_{\mathrm{NL}}=\left(\sigma P_{0}\right)^{-1}$ ( $P_{0}$ - пиковая мошность сигнала) в волокне много больше локальной дисперсионной длины $L_{\mathrm{D}}=T^{2} /\left|\beta_{2}\right|$ ( $T$ - ширина импульса). Более того, можно эффективно устранить член, описываюший линейные потери и накачки, если ввести периодическую функцию $Q(z)$ с дискретными скачками, задаваемую выражением

$$
Q(z)= \begin{cases}\exp \left[-\int_{(k-1) Z_{\mathrm{a}}}^{z} d z^{\prime} \gamma\left(z^{\prime}\right)\right], & (k-1) Z_{\mathrm{a}}<z<k Z_{\mathrm{a}}, \\ 1, & z=k Z_{\mathrm{a}}^{+} .\end{cases}
$$

Теперь с помощью подстановки $E(z, t)=Q(z) U(z, t)$ выражение (1) приводится к виду

$$
i \frac{\partial U}{\partial z}-\frac{1}{2} \beta_{2}(z) \frac{\partial^{2} U}{\partial t^{2}}=i\left\{\sum_{k} \delta\left(z-k Z_{\mathrm{f}}\right)[h(t) *-1]+\sum_{k} \delta\left(z-k Z_{0}\right)\left[f\left(\alpha_{k}^{2}|U|^{2}\right)-1\right]\right\} U
$$

где $\alpha_{k}=Q\left[k Z_{0} \bmod Z_{\mathrm{a}}\right]$ - структурный фактор, учитываюший взаимное расположение усилителей и НОУ. В дальнейшем будем предполагать, что каждое НОУ размешается непосредственно за усилителем, так что можно положить $\alpha_{k}=1$.

Преобразование импульса после прохождения одного сегмента линии передачи можно рассматривать как отображение, переводящее входяший импульс в выходяший. Если взять элемент линии передачи, содержаший НОУ, описываемое уравнением (3), отрезок линейного волокна длины $Z_{0}$ и $m$ фильтров, то отображение сигнала, определенное с точностью до фазового множителя $\mu$, может быть представлено в следуюшем виде:

$$
e^{i \mu} U_{n+1}(t)=\int_{-\infty}^{+\infty} d t^{\prime} K\left(t-t^{\prime} ; Z_{0}\right) f\left(\left|U_{n}\left(t^{\prime}\right)\right|^{2}\right) U_{n}\left(t^{\prime}\right), \quad n=0,1, \ldots
$$

Сигнал здесь берется в точке $n Z_{0}^{-}$на входе НОУ после всех устройств, предшествующих данному НОУ, и ядро отображения $K$ имеет вид

$$
K\left(t-t^{\prime} ; Z_{0}\right)=\frac{1}{2 \pi} \int_{-\infty}^{+\infty} d t^{\prime \prime} H\left(t-t^{\prime}-t^{\prime \prime}\right) F\left(t^{\prime \prime} ; Z_{0}\right),
$$


где $H(t)$ описывает действие фильтров:

$$
H(t)=\mathcal{F}^{-1}\left[\hat{h}^{m}(\omega)\right]
$$

a $F\left(t ; Z_{0}\right)$ представляет собой линейный пропагатор для однородного уравнения, отвечающего уравнению (1):

$$
F\left(t ; Z_{0}\right)=\sqrt{\frac{i}{B_{0}}} e^{-i t^{2} /\left(2 B_{0}\right)}, \quad B_{0}=\int_{n Z_{0}}^{(n+1) Z_{0}} d z \beta_{2}(z) .
$$

В выражении (9) коэффициент $B_{0}$ задает полную совокупную дисперсию. Заметим, что $H(t) \rightarrow \sqrt{2 \pi} \delta(t)$ при $m \rightarrow 0$ (в случае отсутствия фильтров), а $F\left(t ; Z_{0}\right) \rightarrow \sqrt{2 \pi} \delta(t)$ при $B_{0} \rightarrow 0$ (при полной компенсации дисперсии). В дальнейшем для упрошения рассмотрения берутся только гауссовы фильтры, задаваемые функциями

$$
\hat{h}(\omega)=e^{-\omega^{2} /\left(2 \Omega_{\mathrm{f}}^{2}\right)}
$$

где $\Omega_{\mathrm{f}}$ - ширина полосы пропускания. Легко увидеть, что в этом случае ядро $K$ в выражении (7) принимает вид

$$
K\left(t-t^{\prime} ; Z_{0}\right)=\sqrt{G} \sqrt{\frac{i}{2 \pi\left(B_{0}+i m / \Omega_{\mathrm{f}}^{2}\right)}} \exp \left[-\frac{i\left(t-t^{\prime}\right)^{2}}{2\left(B_{0}+i m / \Omega_{\mathrm{f}}^{2}\right)}\right] .
$$

В выражении (11) введено дополнительное усиление $G$, необходимое для того, чтобы скомпенсировать потери энергии сигнала, привносимые в систему НОУ и фильтрами.

С точки зрения переноса сигнала желательно найти (если таковой существует) режим стационарного распространения сигнала, в котором оптический импульс, распространяюшийся вдоль линии передачи, периодически воспроизводится на выходе каждого элемента линии. Это отвечает нахождению стационарной точки отображения (6). Таким образом, чтобы найти форму стационарного импульса $U(t)$, необходимо решить нелинейное интегральное уравнение, получаемое из уравнения (6) при подстановке $U_{n+1}(t)=U_{n}(t)=U(t)$. Когда импульс стационарного состояния оказывается к тому же и устойчивым, всякий исходный сигнал будет монотонно эволюционировать, приближаясь к стационарному состоянию после нескольких отображений.

\section{4. УСТРОЙСТВА СО СТЕПЕННО́Й ЗАВИСИМОСТЬЮ}

В этом разделе анализируется задача отображений, определяемая уравнением (6), когда НОУ представляет собой УСЗ, заданное функцией (3). С помошью пробных функций, описывающих сигнал $U(t)$, можно свести интегральное уравнение отображения в выражении (6) к набору алгебраических уравнений для отображений параметров сигнала. Здесь для описания формы импульса используется гауссова подстановка, а пробная функция выбирается в виде

$$
U(t)=A \exp \left(-\frac{t^{2}}{2 T^{2}}+i C t^{2}+i \xi\right)
$$


где $A=\sqrt{P_{0}}, T, C$ и $\xi$ задают соответственно пиковую амплитуду импульса, его ширину, параметр чирпа и фазовый сдвиг $\left(A, T \in \mathbb{R}^{+}, C, \xi \in \mathbb{R}_{0}\right)$. Отображение (6) сохраняет форму импульса, и подстановка анзаца (12) в уравнение (6) приводит к системе уравнений

$$
\begin{aligned}
A_{n+1} & =\frac{a \sqrt{G} A_{n}^{2 s+1}}{D_{n}^{1 / 4}}, \\
T_{n+1}^{2} & =\frac{D_{n}}{\frac{2 s+1}{T_{n}^{2}}+\frac{m}{\Omega_{\mathrm{f}}^{2}}\left[4 C_{n}^{2}+\frac{(2 s+1)^{2}}{T_{n}^{4}}\right]}, \\
C_{n+1} & =\frac{\left(1-2 B_{0} C_{n}\right) C_{n}-\frac{B_{0}(2 s+1)^{2}}{2 T_{n}^{4}}}{D_{n}},
\end{aligned}
$$

где

$$
D_{N}=\left(1-2 B_{0} C_{n}\right)^{2}+\frac{B_{0}^{2}(2 s+1)^{2}}{T_{n}^{4}}+\frac{m}{\Omega_{\mathrm{f}}^{2}}\left[\frac{4 m C_{n}^{2}}{\Omega_{\mathrm{f}}^{2}}+\frac{2(2 s+1)}{T_{n}^{2}}+\frac{m(2 s+1)^{2}}{\Omega_{\mathrm{f}}^{2} T_{n}^{4}}\right],
$$

$n=0,1, \ldots$. Заметим, что фазовый множитель $\mu$ в отображении (6) выбран таким образом, чтобы скомпенсировать фазовый сдвиг $\left(\xi_{n+1}-\xi_{n}\right)$, возникаюший при движении вдоль элемента линии. Из уравнения (13) видно, что при $m / \Omega_{\mathrm{f}}^{2} \rightarrow 0$ и $B_{0} \rightarrow 0$ допустимым оказывается любое значение параметра чирпа; при этом амплитуда импульса возводится в степень $(2 s+1)$, а ширина импульса, как и следовало ожидать, умножается на $1 / \sqrt{2 s+1}$. Фиксированные точки отображения (13) можно найти аналитически в некоторых выделенных предельных случаях. Интересньй предел возникает, когда линия передачи не содержит фильтров или же их полоса пропускания очень велика, так что $m / \Omega_{\mathrm{f}}^{2} \rightarrow 0$. В этом случае при фиксированных $a, s, B_{0} \neq 0$ и $G$ отображение (13) допускает только одну стационарную точку

$$
\begin{gathered}
A=\left(\frac{2 s+1}{a^{4} G^{2}}\right)^{1 /(8 s)}, \quad T=(2 s+1)^{1 / 4} \sqrt{\left|\frac{B_{0}(s+1)}{s}\right|}, \\
C=-\frac{s}{2 B_{0}(s+1)}, \quad s>-\frac{1}{2}, \quad s \neq 0 .
\end{gathered}
$$

Анализируя линейную устойчивость [9], получим, что стационарная точка (14) оказывается нестабильной при любом $s$. Заметим, что собственные значения линеаризованной системы, связанной с системой (13), зависят от параметра $s$ только через выражения (14). Другой интересный предел системы (13) отвечает случаю, когда дисперсия волокна оказывается полностью скомпенсированной при движении вдоль линии и, тем самым, $B_{0} \rightarrow 0$. В этом случае при фиксированных $a, s, m / \Omega_{\mathrm{f}}^{2} \neq 0$ и $G$ система (13) допускает три решения уравнений для неподвижной точки:

$$
\begin{gathered}
A=\left(\frac{|2 s+1|}{a^{2} G}\right)^{1 /(4 s)}, \quad T=\sqrt{\frac{m(2 s+1)}{2 \Omega_{\mathrm{f}}^{2} s}}, \quad C=0, \quad s<-\frac{1}{2} \quad \text { или } s>0, \\
A=\left(a^{2} G\right)^{1 / 4}, \quad T \geqslant \sqrt{\frac{m}{2 \Omega_{\mathrm{f}}^{2}}}, \quad C= \pm \sqrt{\frac{1}{2 T^{2}}\left(\frac{\Omega_{\mathrm{f}}^{2}}{m}-\frac{1}{2 T^{2}}\right)}, \quad s=-1 .
\end{gathered}
$$


Для неподвижной точки (15) собственные значения линеаризованной системы, порождаемой системой (13), имеют вид

$$
\lambda_{1}=2 s+1, \quad \lambda_{2}=\frac{1}{2 s+1}, \quad \lambda_{3}=\frac{1}{(2 s+1)^{2}} .
$$

Можно непосредственно проверить, что неподвижная точка (15) стабильна при $s=-1$. Собственные значения линеаризованной системы, отвечающей неподвижным точкам (16), зависят от $T$ и от $m / \Omega_{\mathrm{f}}^{2}$, и свойство стабильности для решений (16) может, таким образом, быть исследовано в терминах этих двух параметров.

\section{5. НЕЛИНЕЙНЫЕ ОПТИЧЕСКИЕ ПЕТЛЕОБРАЗНЫЕ ЗЕРКАЛА}

Рассмотрим в этом разделе случай, когда НОУ представляет собой НОПЗ, заданное формулой (3). Читатель может найти описание и принцип действия НОПЗ в работе [10]. Сначала проведем анализ задачи отображения, заданного уравнением (6), для сигналов типа непрерывной волны (НВ). Затем покажем, что стабильное распространение автосолитонов возможно в системах с сильным УД. Это будет показано с помощью непосредственных численных вычислений в рамках основной модели (уравнение (1)). Наконец, применяя вариационный принцип к уравнению (6), мы опишем характеристики стационарного состояния импульса, полученные с помощью численных расчетов.

5.1. Анализ отображения в случае НВ. Выбирая сигнал в виде

$$
U(t) \equiv U_{0}=\sqrt{P_{0}} e^{i \phi}
$$

с постоянными $P_{0}$ и $\phi$, получим из уравнения (6) степенно́е отображение

$$
P_{0, n+1}=a^{2} G \sin ^{2}\left(b P_{0, n}\right) P_{0, n} .
$$

Следует отметить, что НВ-приближение в формуле (18) может быть справедливо лишь в пределе, в котором спектральная ширина импульса много меньше эффективной спектральной ширины системы $\Omega_{\mathrm{eff}}=\sqrt{m /\left[\Omega_{\mathrm{f}}^{2}\left(B_{0}^{2}+m^{2} / \Omega_{\mathrm{f}}^{4}\right)\right]}$. Чтобы при этом сушествовало стабильное стационарное состояние, должны выполняться условия

$$
P_{0, n+1}=P_{0, n}, \quad\left|\frac{\partial P_{0, n+1}}{\partial P_{0, n}}\right|_{P_{0, n+1}=P_{0, n}} \leqslant 1 .
$$

Из условий (20) следуют соотношения

$$
\left(a^{2} G\right)^{-1}=\sin ^{2}\left(b P_{0, n}\right), \quad-1 \leqslant \operatorname{ctg}\left(b P_{0, n}\right) b P_{0, n} \leqslant 0,
$$

которые можно разрешить численно, для того чтобы определить, в каком диапазоне величин $b P_{0, n}$ решение оказывается стабильным. Используемый здесь анализ стабильности с помошью НВ был предложен в работе [10]. Тем не менее этот метод не вполне аккуратен, так как он построен на анализе отображения без учета изменения формы импульса. 
Теперь мы обобщим анализ НВ, включив в него фазовые соображения. Эту задачу можно решать следуюшим образом. Выберем сигнал вида

$$
U(t)=U_{0}+\delta U(t), \quad|\delta U| \ll\left|U_{0}\right|,
$$

с $U_{0}$, заданным формулой (18). Подставим теперь сигнал (22) в отображение (6) и разложим функцию перехода (3) в ряд около $U_{0}$, в результате чего получим

$$
\begin{aligned}
e^{i \mu} U_{0, n+1}= & a \sqrt{G} \sin \left(b P_{0, n}\right) e^{i c P_{0, n}} U_{0, n}, \\
e^{i \mu} \delta U_{n+1}(t)= & a \sin \left(b P_{0, n}\right) e^{i c P_{0, n}} \int_{-\infty}^{+\infty} d t^{\prime} K\left(t-t^{\prime} ; Z_{0}\right) \times \\
& \times\left\{\left[1+\left(b \operatorname{ctg}\left(b P_{0, n}\right)+i c\right) P_{0, n}\right] \delta U_{n}\left(t^{\prime}\right)+\right. \\
& \left.+\left(b \operatorname{ctg}\left(b P_{0, n}\right)+i c\right) P_{0, n} e^{2 i \phi_{n}} \delta U_{n}^{*}\left(t^{\prime}\right)\right\} .
\end{aligned}
$$

Если теперь положить

$$
a \sqrt{G} \sin \left(b P_{0, n}\right)=1, \quad \mu=c P_{0, n},
$$

из выражения (23) получим, что $U_{0, n+1}=U_{0, n}=U_{0}$ есть стационарная точка отображения. Заметим, что первое из соотношений (25) отвечает первому из соотношений (21). Исследование стабильности решения $U_{0}$ можно провести с использованием уравнения (24) и комплексно-сопряженного к нему, с помощью прямых вычислений получив непрерывный спектр собственных значений этой системы в виде

$$
\begin{aligned}
\lambda_{1,2}(\omega) & =e^{-m \omega^{2} /\left(2 \Omega_{\mathrm{f}}^{2}\right)}\left\{\left(1+\operatorname{ctg}\left(b P_{0}\right) b P_{0}\right) \cos \left(\frac{B_{0} \omega^{2}}{2}\right)-c P_{0} \sin \left(\frac{B_{0} \omega^{2}}{2}\right) \pm\right. \\
& \pm\left[\left(\left(c^{2} P_{0}^{2}-\left(1+\operatorname{ctg}\left(b P_{0}\right) b P_{0}\right)^{2}\right) \sin \left(\frac{B_{0} \omega^{2}}{2}\right)-\right.\right. \\
& \left.\left.\left.-2 c P_{0}\left(1+\operatorname{ctg}\left(b P_{0}\right) b P_{0}\right) \cos \left(\frac{B_{0} \omega^{2}}{2}\right)\right) \sin \left(\frac{B_{0} \omega^{2}}{2}\right)+\left(\operatorname{ctg}\left(b P_{0}\right) b P_{0}\right)^{2}\right]^{1 / 2}\right\},
\end{aligned}
$$

$\omega \in[-\infty,+\infty]$, где индексы 1,2 обозначают две ветви спектра. Отметим, что $\lambda_{1,2}(\omega)$ зависят от параметров НОПЗ через отношение $c / b$, где $c / b \in(1,+\infty)$. При фиксированных $B_{0}, m / \Omega_{\mathrm{f}}^{2}, c / b$ и $b P_{0}$ стационарная точка $U_{0}$ стабильна, если выполнено условие $\left|\lambda_{1,2}(\omega)\right| \leqslant 1$ для всех $\omega, i=1,2$. В пределе $B_{0} \rightarrow 0$ нетрудно проверить, что решение $U_{0}$ стабильно для значений величины $b P_{0}$, удовлетворяюших второму из соотношений $(21)$, и при любых $m / \Omega_{\mathrm{f}}^{2}$ и $c / b$. В общем случае, когда $B_{0} \neq 0$, величины $\left|\lambda_{1,2}(\omega)\right|$ можно найти численно и определить область параметров, в которой решение $U_{0}$ стабильно. Отметим, однако, что анализ НВ, который не опирается на форму импульса, не применим при анализе стабильности импульсов конечной шширины, распространяюшихся в системе. Этот анализ можно, тем не менее, провести численно.

5.2. Численный анализ. Примерная схема линии связи, используемая при численном интегрировании уравнения (1), показана на рис. 1. Линия распространения составлена из равного числа сегментов с положительной (аномальной) дисперсией волокна (ПДВ) и с отрицательной (нормальной) дисперсией волокна (ОДВ). Дисперсионное отображение состоит из чередуюшихся блока ПДВ-ОДВ и зеркального блока 


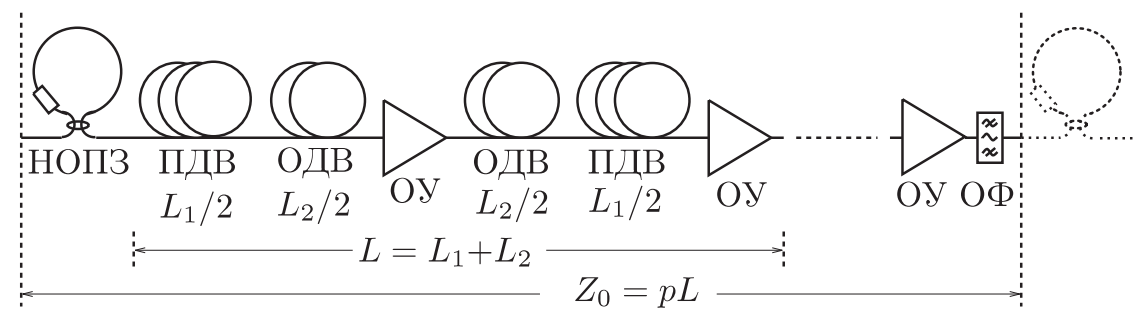

Рис. 1. Базовая секция периодической линии связи.

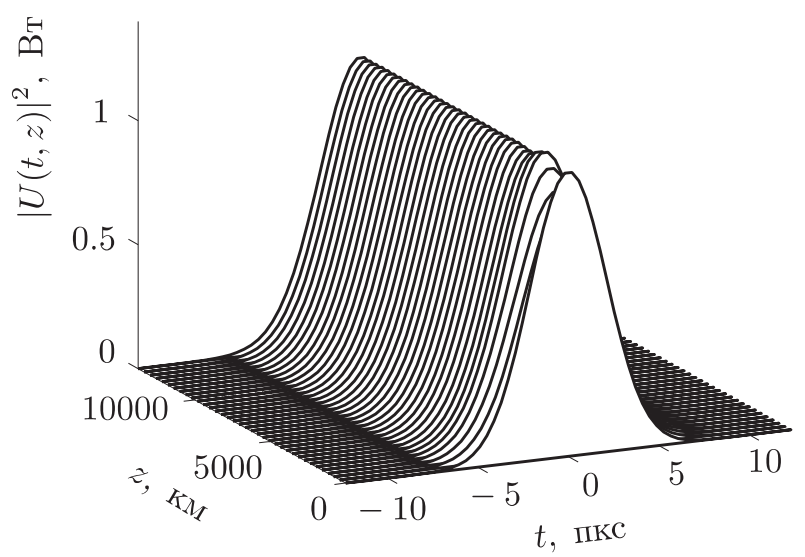

a
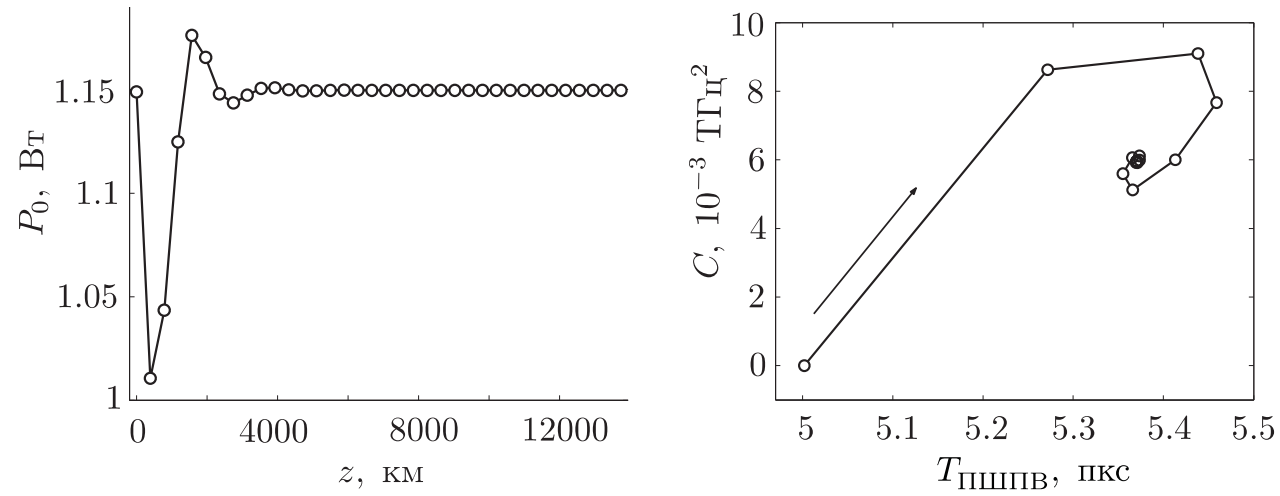

б

Рис. 2. Стробоскопическая эволюция импульса на входе НОПЗ: а) эволюция профиля интенсивности; б) эволюция пиковой мощности импульса $P_{0}$ (слева) и достижение стационарной точки в плоскости ширина импульса - параметр чирпа $C$ (справа).

ОДВ-ПДВ. Для ПДВ и ОДВ были подобраны параметры волокна, имеющие практическую значимость $\left(D_{1}=15 \text { пкс/(нм·км) }, \sigma_{1}=1.84 \text { (В·км }\right)^{-1}, \alpha_{1}=0.22$ дБ $/$ км; $D_{2}=$ -71.2 пкс/(нм·км), $\sigma_{2}=4.28(\mathrm{~B} \cdot \text { 'км })^{-1}, \alpha_{2}=0.65$ дБ/км) [6]. Отметим, что при вы- 
числениях учитьвалась нелинейность волокна. Оптический усилитель (ОУ), который компенсировал потери в волокне, помешался непосредственно за каждым из двух блоков. Высокие значения локальной дисперсии волокна при малых значениях длительности импульсов, обычно используемых для работы с системами при высоких скоростях передачи данных, выходят за пределы области сушествования решений в виде УД-солитонов [4]. НОПЗ используются через целое число $p$ периодов дисперсионного отображения, $Z_{0}=p L$. Отметим, что в этом случае $B_{0}=\left\langle\beta_{2}\right\rangle Z_{0}=-\lambda^{2}\langle D\rangle Z_{0} /\left(2 \pi c_{0}\right)$ (через $\langle\cdot\rangle$ обозначено усреднение на периоде компенсации дисперсии $L)$. В конфигурации, рассматриваемой нами, $Z_{0}=391$ км и $p=5$. Единственный $(m=1)$ гауссов оптический фильтр (ОФ) помешается за усилителем, но перед НОПЗ. В качестве примера рассматривалась конфигурация НОПЗ с несбалансированными потерями и использовалось предварительное усиление входящих в НОПЗ импульсов (см. детали в работе [6]). Параметры $a, b$ и $c$ функции перехода (3) принимают соответственно значения $0.06373,1.823$ и $1.839 \mathrm{Bт}^{-1}$. НОПЗ при этом действует как насышаемьй поглотитель [10] и потому отфильтровывает излучение низкой интенсивности от высокоэнергетических импульсов. Это позволяет восстановить амплитуду и форму импульса [5], [6]. Следуя работе [6], система используется таким образом, чтобы пиковая мошность импульсов в стационарном состоянии (если такое сушествует) лежала бы в области, расположенной за первым пиком мошностной НВ-характеристики НОПЗ. В этой области НОПЗ обеспечивает управление амплитудой импульсов с помошью отрицательной обратной связи [10], что может обеспечить стабилизацию флуктуаций амплитуды импульсов [6]. Разумеется, такого рассуждения недостаточно для обеспечения стабильного прохождения импульсов через систему, и мы используем его здесь только для уменьшения размерности пространства параметров системы, при которых ведется поиск стационарных локализованных состояний. На рис. 2 профиль импульса показан стробоскопически в точках входа в НОПЗ. В этом примере в систему вводится нечирпированный гауссов импульс с пиковой мошностью $P_{0}=1.15 \mathrm{~B}$ т (отвечаюшей мошности $3.5 \mathrm{MB}$ в начальной точке трансмиссии) и с шириной импульса ПШПВ (полной шириной на половинной высоте) $T_{\text {Пшпв }}=5$ пкс. Параметры системы $\langle D\rangle=0.009$ пкс $/\left(\right.$ нм'км),$\quad \delta \nu_{\mathrm{f}}=\sqrt{\ln 2} \Omega_{\mathrm{f}} / \pi=0.1$ Тгц и $G=627.0$ $(28.0$ дБ). Параметр чирпа импульса вычисляется как

$$
C=\frac{\operatorname{Im} \int_{-\infty}^{+\infty} d t U^{2}\left(U_{t}^{*}\right)^{2}}{\int_{-\infty}^{+\infty} d t|U|^{4}} .
$$

Из рис. 2 видно, что импульс переходит в свое стационарное состояние в течение достаточно короткого начального отрезка пути. Этот результат демонстрирует осушествимость режима стационарного распространения импульса в системе и показывает, что использование встроенных НОПЗ позволяет перевести режим квазилинейной передачи сигнала в режим автосолитонного распространения [6]. Отметим, что ту же стробоскопическую картину, что и на рис. 2 , можно получить, просто итерируя уравнение отображения (6). Мы также определили допустимые пределы ширины полосы пропускания фильтра и усредненной по пути дисперсии волокна, при которых возможен стационарный режим прохождения импульса. Результаты показаны на рис. 3, где переменные $\delta \nu_{\mathrm{f}}$ и $\langle D\rangle$ принимают значения в области величин, используемых на практике. Величина $G$ 
на рис. 3 выбрана таким образом, чтобы пиковая мощность стационарного состояния импульса была примерно 1.15 Вт на входе НОПЗ.

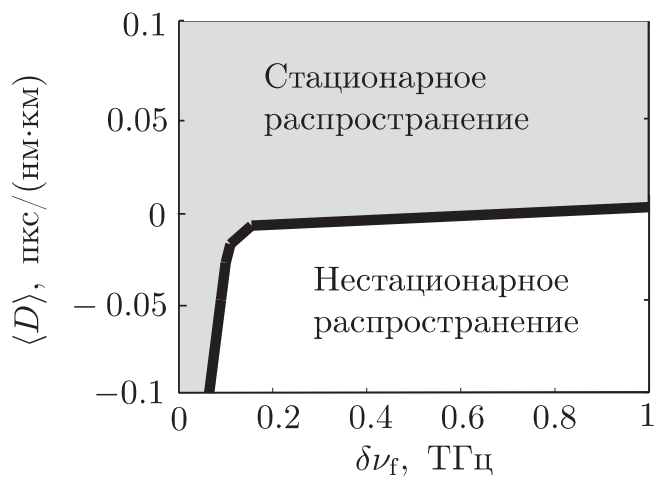

Рис. 3. Границы зоны, в которой возможен режим стационарного распространения импульса в плоскости спектральная ширина фильтра $\delta \nu_{\mathrm{f}}-$ усредненная дисперсия $\langle D\rangle$.

5.3. Вариационный подход и сравнение с численным расчетом. Как было указано в разделе 3 , для нахождения режима стационарного состояния прохождения импульса необходимо решить интегральное уравнение отображения (6) для стационарных точек. Однако аналитическое решение задачи весьма затруднительно при использованиии НОПЗ ввиду наличия специфической нелинейной функции, описывающей прохождение через НОПЗ. Поэтому здесь применяется упрощенный вариационный подход. Из численного анализа следует, что стационарное состояние формы импульса на входе НОПЗ всегда можно хорошо аппроксимировать с помощью гауссова профиля. Поэтому пробный входящий импульс $U(t)$ для отображения можно выбрать в виде гауссова импульса с пиковой мошностью $P_{0}$ (до поры не определенной), среднеквадратичной $(\mathrm{CK})$ шириной импульса $T_{\mathrm{CK}}$ и $\mathrm{CK}$-параметром чирпа $C_{\mathrm{CK}}$ :

$$
U_{n}(t)=\sqrt{P_{0}} \exp \left(-\frac{t^{2}}{4 T_{\mathrm{CK}}^{2}}+i C_{\mathrm{CK}} t^{2}\right) .
$$

На выходе отображения, задаваемого уравнением (6), форма импульса $U_{n+1}(t)$ будет в обшем случае негауссовой, но он будет иметь форму, близкую к гауссовой, и зависеть от параметров входяшего сигнала, $U_{n+1}=U_{n+1}\left(t ; P_{0}, T_{\mathrm{CK}}, C_{\mathrm{CK}}\right)$. Потребуем теперь, чтобы пиковая мощность, ширина импульса и чирп выходящего сигнала совпали с соответствуюшими характеристиками входящего гауссова сигнала. Это задает систему трансцендентных уравнений для искомых параметров $P_{0}, T_{\mathrm{CK}}$ и $C_{\mathrm{CK}}$ :

$$
\begin{aligned}
P_{0} & =\frac{1}{\sqrt{2 \pi} T_{\mathrm{CK}}} \int_{-\infty}^{+\infty} d t\left|U_{n+1}\left(t ; P_{0}, T_{\mathrm{CK}}, C_{\mathrm{CK}}\right)\right|^{2}, \\
T_{\mathrm{CK}}^{2} & =\frac{\int_{-\infty}^{+\infty} d t t^{2}\left|U_{n+1}\left(t ; P_{0}, T_{\mathrm{CK}}, C_{\mathrm{CK}}\right)\right|^{2}}{\int_{-\infty}^{+\infty} d t\left|U_{n+1}\left(t ; P_{0}, T_{\mathrm{CK}}, C_{\mathrm{CK}}\right)\right|^{2}},
\end{aligned}
$$




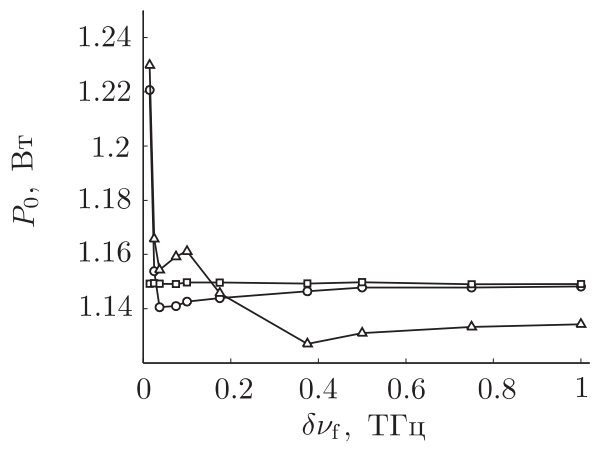

a

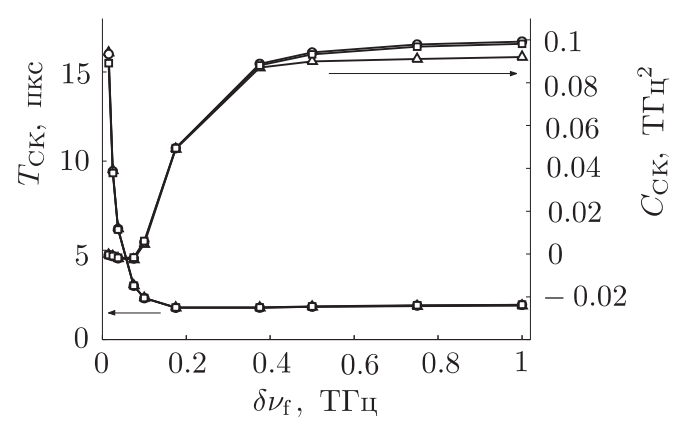

6

Рис. 4. Характеристики стационарного состояния: а) пиковая мощность импульса $P_{0}$, б) CK-ширина импульса $T_{\mathrm{CK}}$ (левая ось) и $\mathrm{CK}$-параметр чирпа $C_{\mathrm{CK}}$ (правая ось) на входе НОПЗ в зависимости от спектральной ширины фильтра; о - отображение, $\triangle$ - вариационная модель, $\square$ - полный численный расчет.

$$
C_{\mathrm{CK}}=\frac{\operatorname{Im} \int_{-\infty}^{+\infty} d t U_{n+1}^{2}\left(t ; P_{0}, T_{\mathrm{CK}}, C_{\mathrm{CK}}\right)\left(\partial_{t} U_{n+1}^{*}\left(t ; P_{0}, T_{\mathrm{CK}}, C_{\mathrm{CK}}\right)\right)^{2}}{\int_{-\infty}^{+\infty} d t\left|U_{n+1}\left(t ; P_{0}, T_{\mathrm{CK}}, C_{\mathrm{CK}}\right)\right|^{4}}
$$

Если сушествует решение системы (28), то оно задает вариационное приближение для параметров стационарного состояния импульса. В частности, в качестве приближения для формы стационарного состояния импульса можно использовать гауссов анзац с найденными значениям величин $P_{0}, T_{\mathrm{CK}}$ и $C_{\mathrm{CK}}$. Предсказания вариационной модели (уравнения $(27),(28))$ и результат итеративного численного интегрирования уравнения отображения (6) сравнивались с результатами численного расчета. На рис. 4 изображены пиковая мошность, CK-ширина и СK-параметр чирпа для стационарного состояния импульса как функции спектральной ширины фильтра при $\langle D\rangle=0.009$ пкс/(нм·км), и те же значения $G$ использованы на рис. 3. Результаты обнаруживают хорошее согласие, особенно для ширины импульса и для параметра чирпа. Что касается амплитуды импульса, то для нее расхождения между решениями уравнения (6) и численными расчетами, наблюдаемые при малых величинах $\delta \nu_{\mathrm{f}}$ (имеюшие максимальный порядок $6 \%$ ), могут быть связаны с тем, что при малых спектральных ширинах фильтров эффект нелинейности в передаточном волокне, которым пренебрегалось в модели (6), становится достаточно значимым. При бо́льших $\delta \nu_{\mathrm{f}}$ расхождение между предсказаниями модели (6) и численными расчетами не превосходит $0.6 \%$, что полностью подтверждает предположение о линейности, лежашее в основе уравнения (6) в данной области спектральных ширин фильтров. Отклонение предсказаний вариационной модели как от численных расчетов, так и от предсказаний модели (6) не превосходит $2 \%$ в области спектральных ширин, в которой предположение о линейности полностью обоснованно. Профиль интенсивности стационарного состояния и чирп (первая производная по времени от фазы) импульса на входе НОПЗ изображены на рис. 5 при $\delta \nu_{\mathrm{f}}=0.1$ ТГц. Результаты вариационной модели находятся в хорошем согласии с численными расчетами и результатами уравнения (6) в центральной части импульса. 


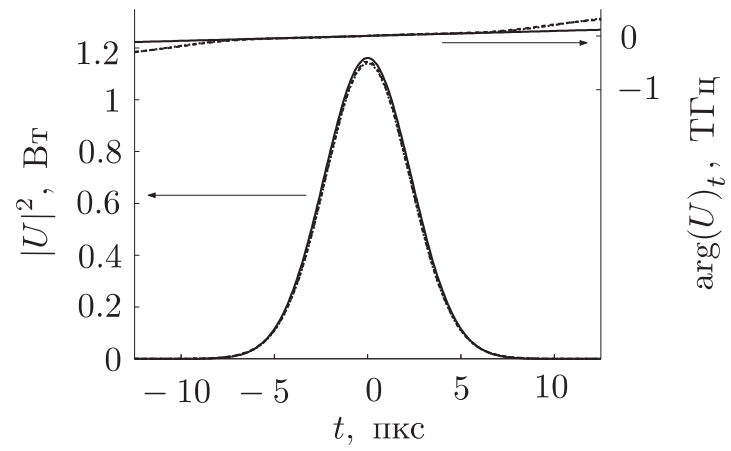

Рис. 5. Интенсивность $|U|^{2}$ (левая ось) и чирп $\arg (U)_{t}$ (правая ось) стационарного состояния импульса на входе НОПЗ. Пунктирная линия - отображение, сплошная линия вариационная модель, штриховая линия - численный расчет.

\section{6. ЗАКЛЮЧЕНИЕ}

Нами была представлена теоретическая модель, описывающая прохождение импульса в волоконных системах в квазилинейном режиме с НОУ, периодически встроенными в линию передачи. В случае применения НОПЗ было численно показано, что в такой системе можно наблюдать образование автосолитонов. Характеристики стационарного распространения импульса определялись также с помощью вариационного принципа; оказалось, что результаты теоретического анализа воспроизводят результаты численных расчетов с достаточно хорошей точностью.

Благодарности. Эта работа была частично поддержана NATO Collaborative Linkage Program (№ PST.CLG.980068).

\section{Список литературы}

[1] A. Hasegawa, Y. Kodama. Opt. Lett. 1990. V. 15. P. 1443-1445.

[2] I. Gabitov, S. K. Turitsyn. Opt. Lett. 1996. V. 21. P. 327-329.

[3] N. J. Smith, F. M. Knox, N. J. Doran, K. J. Blow, I. Bennion. Electron. Lett. 1996. V. 32. P. $54-55$.

[4] J. H. B. Nijhof, N. J. Doran, W. Forysiak, A. Berntson. Electron. Lett. 1998. V. 34. P. 481-482.

[5] S. Boscolo, J. H. B. Nijhof, S. K. Turitsyn. Opt. Lett. 2000. V. 25. P. 1240-1242.

[6] S. Boscolo, S. K. Turitsyn, K. J. Blow. IEEE Photon Lett. 2002. V. 14. P. 30-32.

[7] D. Rouvillain, P. Brindel, E. Seguineau, L. Pierre, O. Leclerc, H. Choumane, G. Aubin, J. L. Oudar. Electron. Lett. 2002. V. 38. P. 1113-1114.

[8] Б. С. Кернер, В. В. Осипов. Автосолитоны. Наука: М., 1991.

[9] J. D. Logan. Applied Mathematics: A Contemporary Approach. N.Y.: John Wiley \& Sons, 1987.

[10] N. J. Smith, N. J. Doran. J. Opt. Soc. Am. B. 1995. V. 12. P. 1117-1125. 